\title{
BMJ Open Prognostic impact of alkaline phosphatase for in-hospital mortality in patients with acute coronary syndrome: a prospective cohort study in China
}

Tongtong Yu, Yundi Jiao, Jia Song, Dongxu He, Jiake Wu, Zongyu Wen, Na Sun, Weili Duan, Zhijun Sun, Zhaoqing Sun

To cite: Yu T, Jiao Y, Song J, et al. Prognostic impact of alkaline phosphatase for in-hospital mortality in patients with acute coronary syndrome: a prospective cohort study in China. BMJ Open 2019;9:e025648. doi:10.1136/ bmjopen-2018-025648

- Prepublication history and additional material for this paper are available online. To view these files, please visit the journal online (http://dx.doi. org/10.1136/bmjopen-2018025648).

Received 26 July 2018 Revised 19 March 2019 Accepted 09 July 2019
Check for updates

(C) Author(s) (or their employer(s)) 2019. Re-use permitted under CC BY-NC. No commercial re-use. See rights and permissions. Published by BMJ.

Department of Cardiology, Shengjing Hospital of China Medical University, Shenyang, China

Correspondence to Dr Zhaoging Sun; sunzhaoqing@vip.163.com

\section{ABSTRACT}

Objectives Alkaline phosphatase (ALP) can promote vascular calcification, but the association between ALP and in-hospital mortality in patients with acute coronary syndrome (ACS) is not well defined.

Design A prospective cohort study.

Setting and participants A total of 6368 patients with ACS undergoing percutaneous coronary intervention (PCI) from 1 January 2010 to 31 December 2017 were analysed.

Main outcome measures In-hospital mortality was used in this study.

Results ALP was analysed both as a continuous variable and according to three categories. After multivariable adjustment, in-hospital mortality was significantly higher in Tertile 3 group (ALP > 85 U/L) (OR: 2.399, 95\% Cl 1.080 to 5.333, $p=0.032$ ), compared with other two groups (Tertile 1: < $66 \mathrm{U} / \mathrm{L}$; Tertile 2: 66-85 U/L). When ALP was evaluated as a continuous variable, after multivariable adjustment, the ALP level was associated with an increased risk of in-hospital mortality (OR: $1.011,95 \% \mathrm{Cl}$ 1.002 to $1.020, p=0.014)$. C-statistic of ALP for predicting in-hospital mortality was $0.630(95 \% \mathrm{Cl} 0.618$ to 0.642 , $\mathrm{p}=0.001$ ). The cut-off value was $72 \mathrm{U} / \mathrm{L}$ with a sensitivity of 0.764 and a specificity of 0.468 . However, ALP could not significantly improve the prognostic performance of Global Registry of Acute Coronary Events (GRACE) score (GRACE score+ALP vs GRACE score: C-statistic: $z=0.485, p=0.628$; integrated discrimination improvement: $0.014, p=0.056$; net reclassification improvement: $0.020, p=0.630$ ). Conclusions In patients with ACS undergoing PCI, ALP was an independent predictor of in-hospital mortality. But it could not improve the prognostic performance of GRACE score.

\section{INTRODUCTION}

Patients with acute coronary syndrome (ACS) usually surfer a poor prognosis. ${ }^{1-4}$ The timely prognostic evaluation and adequate clinical treatment is important for the improvement of the prognosis in patients with ACS. Coronary artery calcium contributes to atherosclerosis, and is also a predictor of adverse cardiovascular events. ${ }^{5-7}$ Inorganic pyrophosphate
Strengths and limitations of this study

- This study explored the association between alkaline phosphatase (ALP) and in-hospital mortality in 6368 acute coronary syndrome patients undergoing percutaneous coronary intervention.

- This study also confirmed whether ALP could improve the prognostic performance of Global Registry of Acute Coronary Events score.

- In this study, data on serum parathyroid hormone level, serum vitamin D level and dietary vitamin D intake was not obtained, which might affect ALP levels.

- In this study, coronary calcification was assessed by angiography, which might underestimate the frequency and degree of coronary calcium.

is an inhibitor of vascular calcification. As a membrane-bound metallo enzyme, alkaline phosphatase (ALP) catalyses inorganic pyrophosphate and may promote vascular calcification. ${ }^{8}$ Some studies found that elevated serum ALP was associated with the coronary artery calcium score assessed by CT scanning and the severity of coronary artery disease (CAD).${ }^{910}$ Other studies confirmed the association between higher ALP and the long-term adverse outcomes in patients with $\mathrm{CAD},{ }^{11-13}$ $\mathrm{ACS}^{1415}$ and ST-segment elevation myocardial infarction (STEMI) ${ }^{1617}$ However, the role of ALP level in predicting in-hospital mortality in patients with ACS is not clearly defined.

The Global Registry of Acute Coronary Events (GRACE) score can predict in-hospital mortality in ACS and helps us discriminate high-risk patients. ${ }^{1-4}$ However, new markers are not included in GRACE score, such as ALP.

In this study, we aimed to assess whether ALP was a useful clinical parameter to predict in-hospital mortality in patients with ACS undergoing percutaneous coronary intervention (PCI). We 
also confirmed whether ALP could improve the prognostic performance of GRACE score.

\section{METHODS}

\section{Study design and setting}

This prospective cohort study took place at a large-scale hospital in Northeast China (Shengjing Hospital of China Medical University, Shenyang, China). The consecutive patients with ACS undergoing PCI at from 1 January 2010 to 31 December 2017 were eligible for this study. ACS was defined according to the current guidelines. ${ }^{1-4}$ PCI was performed in accordance with the current guidelines. ${ }^{1-4}$ Clinical data and procedural data of all cases were gained by the investigators using a uniform questionnaire. GRACE score were determined as defined previously. ${ }^{1-4}$ In all cases, venous blood samples were drawn on admission in standard tubes. Tissue-non-specific ALP and serum creatinine were measured by a completely automated biochemistry-immunity analyser (Ci 16200, Abbott, USA) in the core laboratory of Shengjing Hospital. The reference interval of ALP in our laboratory was 40-150 U/L. In-hospital death was determined as defined previously. ${ }^{18}$ Exclusion criteria included (1) known hepatic, biliary or bone disease (114 cases); (2) excessive alcohol consumption (112 cases); (3) chronic kidney failure with dialysis on admission (89 cases); (4) no ALP or GRACE score data (72 cases). Finally, the study included 6368 patients with ACS undergoing PCI. The mean duration of hospitalisation was $7.5 \pm 4$.4 days. Figure 1 represents the flowchart of patient selection. All individuals

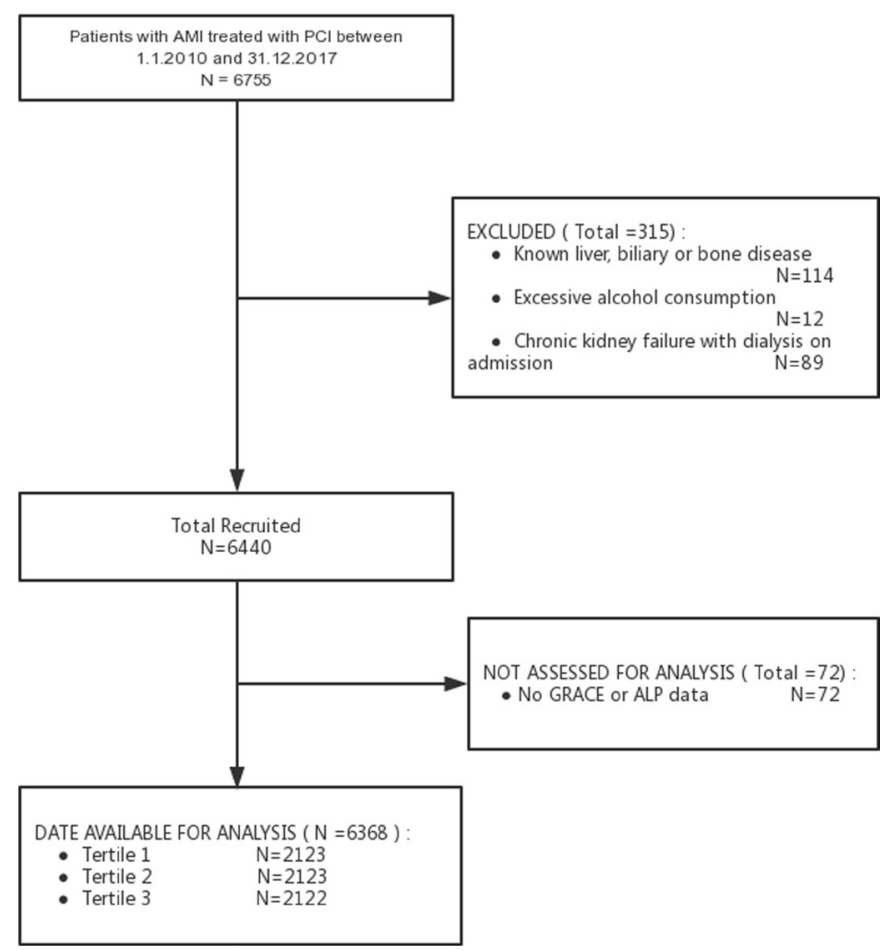

Figure 1 Flow diagram of participant selection. ALP, alkaline phosphatase; AMI, Acute Myocardial Infarction; GRACE, Global Registry of Acute Coronary Events; PCI, percutaneous coronary intervention. were divided into three groups according to the tertile of ALP level on admission (Tertile 1: <66 U/L ( $\mathrm{n}=2123)$; Tertile 2: 66-85U/L (n=2123); Tertile 3: $>85 \mathrm{U} / \mathrm{L}$ $(\mathrm{n}=2122))$. This study complies with the Declaration of Helsinki, and Shengjing Hospital of China Medical University Research Ethics Committee approved the research protocol. Written informed consent was formally obtained from all participants.

\section{Patient and public involvement}

No patients or public were involved in the present study.

\section{Statistical analysis}

Quantitative variables with normal distribution were represented as mean $\pm \mathrm{SD}$ and compared with Kruskal-Wallis H-test. Quantitative variables without normal distribution were represented as median (IQR) and compared with Kruskal-Wallis H-test. Categorical variables are presented as counts and proportions (\%) and compared with $\chi^{2}$ test. Logistic univariate regressions were performed to evaluate predictors of in-hospital death using 'Enter' (online supplementary appendices S1-S3). A multivariate logistic regression model was used to identify independent predictors of in-hospital death using 'Forward: conditional'. Variables with $\mathrm{p}<0.05$ in univariate analysis were entered a multivariate analysis. ALP was analysed as a continuous variable and categories variable, respectively. Results were reported as ORs with associated 95\% CIs. The predictive performance of ALP, GRACE score and GRACE score +ALP was assessed by indices of discrimination (C-statistic), calibration (the Hosmer-Lemeshow test, the Nagelkerke- $\mathrm{R}^{2}$ ) and precision (Brier scores). The C-statistic, which was defined by the area under the receiver operating characteristic (ROC-AUC) curve in relation to in-hospital mortality, was compared using a non-parametric test developed by DeLong et al. ${ }^{19}$ C-statistic values > 0.5, 0.75, 0.93 were considered as fair, good, very good accuracy. Each model was entered into a logistic regression model to get the individual risk probability of in-hospital death, respectively. The Hosmer-Lemeshow (HL) test and the Nagelkerke- $\mathrm{R}^{2}$ from the regression modelling were used as indicators of goodness-of-fit of each risk model and to assess their calibration ability. ${ }^{20}$ The Brier scores of ALP, GRACE score and GRACE score +ALP were also calculated. ${ }^{21}$ Lower Brier scores indicated better precision. ${ }^{21}$ We also used the absolute integrated discrimination improvement (IDI) and category-free net reclassification improvement (NRI) to evaluate improvements in risk prediction quantisation of ALP and GRACE score $+\mathrm{ALP} 0 .{ }^{22}$ All tests were two-sided, and the statistical significance was defined as $\mathrm{p}<0.05$. All statistical analyses were performed using the Statistical Analysis System V.9.4.

\section{RESULTS}

\section{Participants and baseline characteristics}

Figure 1 represents the flowchart of patient selection. The final study cohort consisted of 6368 patients with ACS undergoing PCI. Clinical characteristics were 
shown in table 1 . Tertile 3 group had significantly higher percentages of females, STEMI and moderate or severe calcification, compared with other groups. Tertile 3 group also had a tendency towards increasing systolic blood pressure, heart rate, Troponin-I, albumin, serum glutamic oxaloacetic transaminase (SGOT), serum glutamate-pyruvate transaminase (SGPT), haemoglobin, leucocyte count and platelet count on admission. There was a significant trend of decreasing creatinine in Tertile 3 group. The percentage of thrombolysis in myocardial infarction (TIMI) flow grade $0 / 1$ was significantly lower in Tertile 3 group. Tertile 3 group also had the trend to

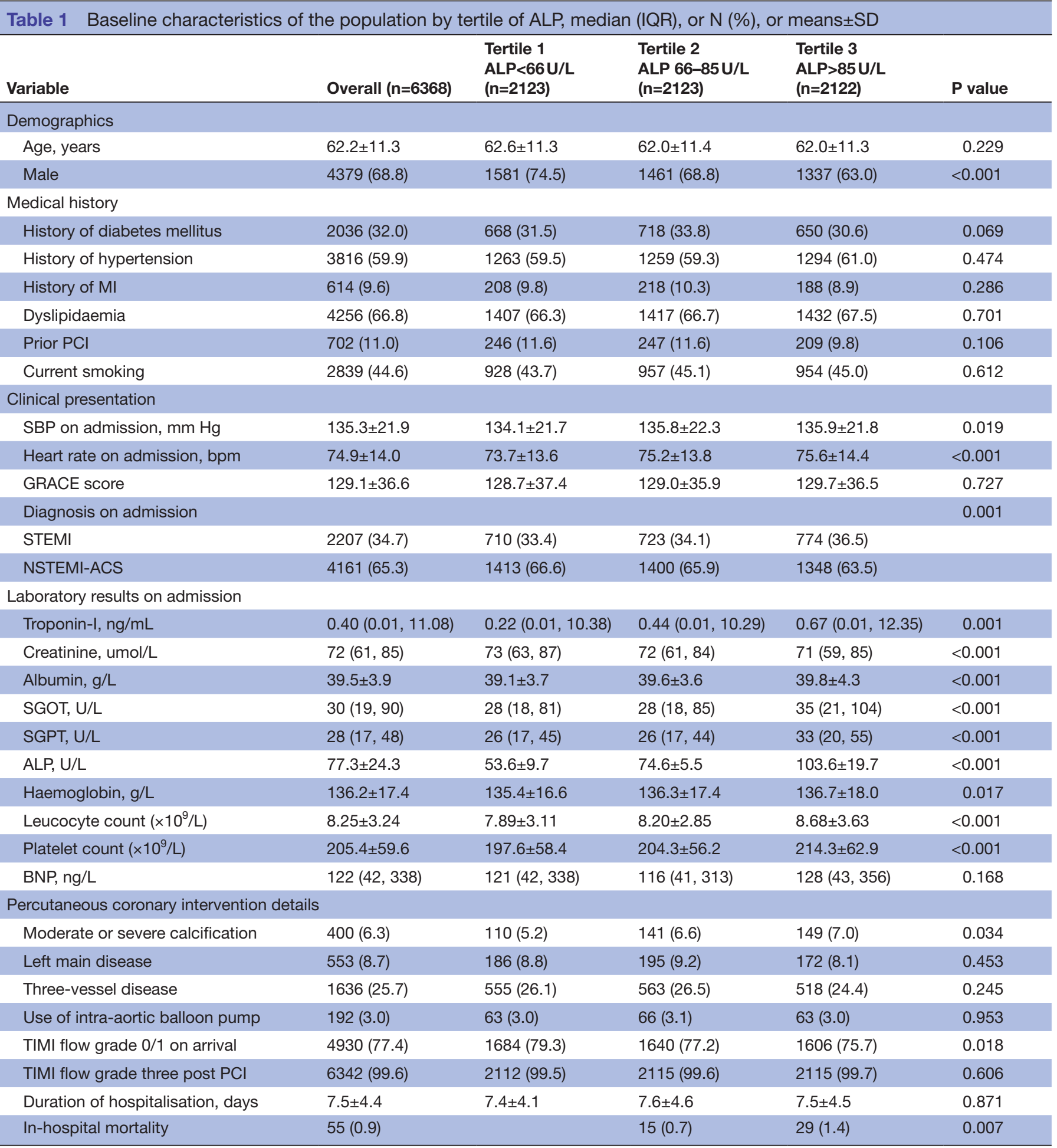

ALP, alkaline phosphatase; BNP, brain natriuretic peptide;MI, myocardial infarction; NSTEMI-ACS, non-ST-segment elevation myocardial infarction of acute coronary syndrome; PCl, percutaneous coronary intervention; SBP, systolic blood pressure; SGOT, serum glutamic oxaloacetic transaminase; SGPT, serum glutamate-pyruvate transaminase; STEMI, ST-segment elevation myocardial infarction;TIMI, thrombolysis in myocardial infarction; bpm, beats per minute. 
Table 2 Effects of multiple variables on clinical outcomes in univariate and multivariate analysis

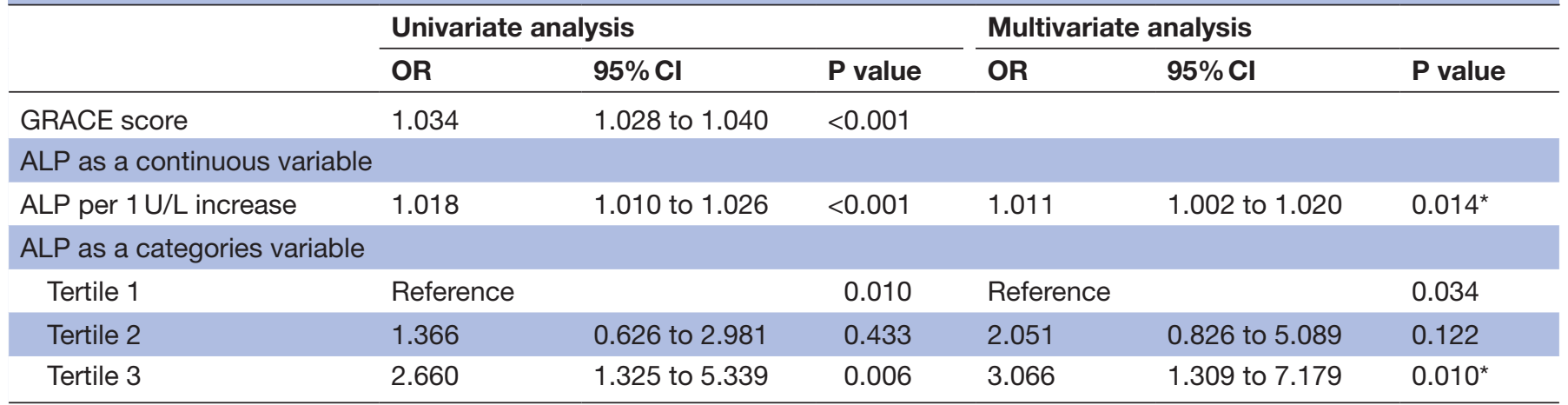

*Adjusted for age, SBP on admission, heart rate on admission, diagnosis on admission, Troponin-I, creatinine, albumin, SGOT, SGPT, haemoglobin, leucocyte count, BNP, moderate or severe calcification, left main disease, three-vessel disease, use of intra-aortic balloon pump and TIMI flow grade three post-PCI.

ALP, alkaline phosphatase; PCI, percutaneous coronary intervention; SGOT, serum glutamic oxaloacetic transaminase; SGPT, serum glutamate-pyruvate transaminase.

suffer a higher in-hospital mortality $(1.4 \%$ vs $0.5 \%$ and $0.7 \%, \mathrm{p}=0.007$ ) (table 1).

\section{Prognostic performance of ALP in prognosis prediction}

The univariate analysis in online supplementary appendices $\mathrm{S} 1$ found that many variables had significant effects on in-hospital mortality: age, systolic blood pressure on admission, heart rate on admission, diagnosis on admission, Troponin-I, creatinine, albumin, SGOT, SGPT, haemoglobin, leucocyte count, brain natriuretic peptide, moderate or severe calcification, left main disease, threevessel disease, use of intra-aortic balloon pump and TIMI flow grade three post PCI (online supplementary appendices S1).

The univariate analysis revealed that ALP was associated with in-hospital mortality (OR: 1.018, 95\% CI 1.010 to $1.026, \mathrm{p}<0.001$, for per $1 \mathrm{U} / \mathrm{L}$ increase) (table 2). After adjusting for covariates, the association was still present: an increased in-hospital mortality risk of $1.1 \%$ for per $1 \mathrm{U} / \mathrm{L}$ increase in ALP concentration (OR: 1.011, 95\% CI 1.002 to $1.020, \mathrm{p}=0.014$ ) (table 2).

When categorised into three groups, ALP still significantly predicted in-hospital mortality (table 2). In the univariate analysis, compared with Tertile 1 and 2 group, Tertile 3 group had a substantially higher risk of in-hospital death (OR: 2.660, 95\% CI 1.325 to 5.339, p<0.001) (table 2). In the multivariable analysis, Tertile 3 group still conferred a significantly higher in-hospital mortality (OR: $3.066,95 \%$ CI 1.309 to $7.179, \mathrm{p}=0.010$ ) (table 2 ).
The C-statistic of ALP, GRACE score and GRACE score +ALP in predicting in-hospital mortality were 0.630 (95\% CI 0.618 to 0.642$), 0.842$ (95\% CI 0.833 to 0.851 ) and 0.847 (95\% CI 0.838 to 0.856 ) (table 3 and figure 2 ), respectively. The cut-off value for ALP was $72 \mathrm{U} / \mathrm{L}$ with a sensitivity of 0.764 and a specificity of 0.468 .

The study cohort was also divided into two subgroup: STEMI group (STEMI group) and non-STEMI of ACS group (NSTEMI-ACS group). The subgroup analysis was then taken. In STEMI group, ALP was associated with in-hospital mortality in the univariate or multivariable analysis, when ALP was analysed both as a continuous variable and according to three categories (online supplementary appendices S4). The same conclusion was also got in NSTEMI-ACS group (online supplementary appendices S5).

\section{Improvement of the prognostic performance of grace score + ALP}

Table 3 presented that the HL p value and the Nagelkerke- $\mathrm{R}^{2}$ of GRACE score was the highest; the Brier score of GRACE +ALP was the lowest. However, the new model in which the GRACE score was combined with ALP could not improve the prognostic performance. The prognostic performance of GRACE +ALP was similar to that of GRACE score (C-statistic: $\mathrm{z}=0.485$, $\mathrm{p}=0.628$; IDI: 0.014, $\mathrm{p}=0.056$; NRI: $0.020, \mathrm{p}=0.630$ ) (table 4). In the subgroup analysis, the prognostic performance of GRACE +ALP was also similar to that of GRACE score in STEMI group

Table 3 GRACE, GRACE+ALP and ALP performance for the prognosis prediction

\begin{tabular}{|c|c|c|c|c|c|c|c|}
\hline & \multicolumn{4}{|c|}{ Discrimination } & \multicolumn{2}{|l|}{ Calibration } & \multirow{2}{*}{$\begin{array}{l}\text { Precision } \\
\text { Brier score }\end{array}$} \\
\hline & C-statistic & SE & $P$ value & $95 \% \mathrm{Cl}$ & HL $p$ value & $\mathbf{R}^{2}$ & \\
\hline GRACE & 0.842 & 0.0276 & $<0.001$ & 0.833 to 0.851 & 0.806 & 0.186 & 0.0082 \\
\hline ALP & 0.630 & 0.0412 & 0.001 & 0.618 to 0.642 & 0.496 & 0.027 & 0.0085 \\
\hline GRACE+ALP & 0.847 & 0.0290 & $<0.001$ & 0.838 to 0.856 & $<0.001$ & 0.102 & 0.0081 \\
\hline
\end{tabular}

ALP, alkaline phosphatase; GRACE, Global Registry of Acute Coronary Events; HL, Hosmer-Lemeshow. 


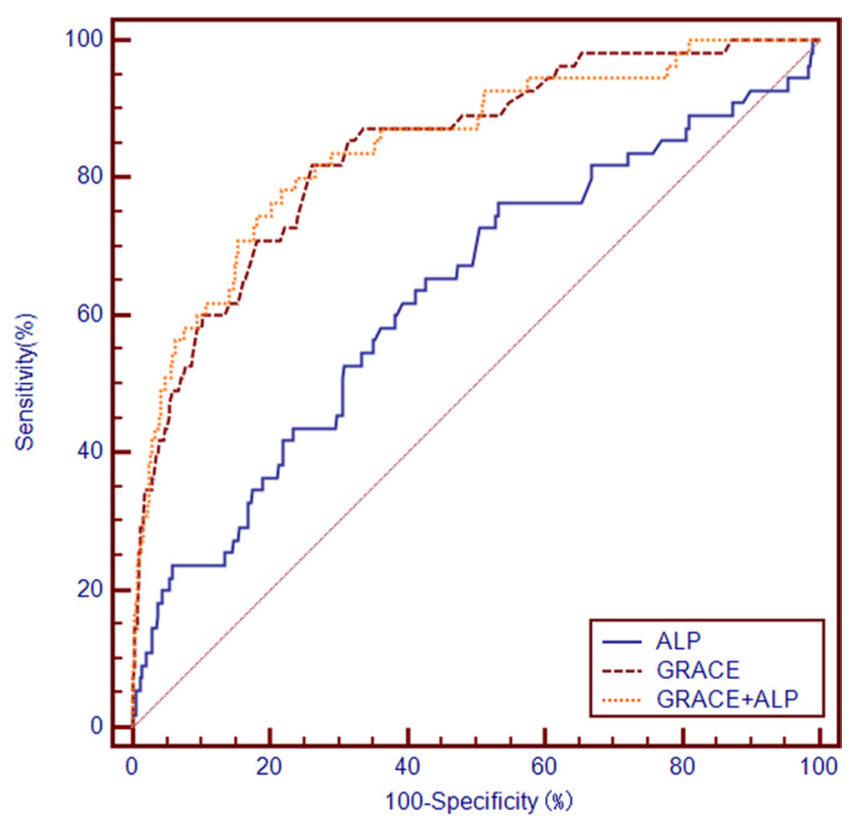

Figure 2 Receiver operating characteristic curves of ALP, grace and GRACE +ALP for in-hospital death prediction. ALP,alkaline phosphatase; GRACE, GlobalRegistry of Acute Coronary Events.

(online supplementary appendices S6) or NSTEMI-ACS group (online supplementary appendices $\mathrm{S} 8$ ).

\section{DISCUSSION}

The present study tested the association between ALP and in-hospital mortality in patients with ACS undergoing PCI. The main findings were as follows: (1) ALP was an independent predictor of in-hospital mortality; (2) the prognostic performance of GRACE score +ALP was similar to GRACE score, and ALP could not improve the prognostic performance of the original GRACE score model.

At first, an independent association was confirmed between ALP and the risk of adverse outcomes in patients with normal kidney function or kidney failure. ${ }^{23}{ }^{24}$ Then, meta-analyses found the association between ALP and the incident of cardiovascular disease. ${ }^{2526}$ Tonelli et al first studied the prognostic value of ALP for the prognosis in survivors of myocardial infarction. ${ }^{11}$ They found that ALP was an independent predictor of adverse outcomes among 4115 participants with a previous myocardial infarction. ${ }^{11}$ This observation then was verified and extended by other researches that elevated ALP was an independent predictor of adverse outcomes in patients with $\mathrm{CAD},{ }^{1213} \mathrm{ACS}^{1415}$ and STEMI. ${ }^{1617}$ However, no study focuses on the prognostic value of ALP for in-hospital mortality in patients with ACS undergoing PCI. This study demonstrated that, in patients with ACS undergoing PCI, ALP was associated with in-hospital mortality. With ALP as a continuous variable, we found that the risk of in-hospital mortality increased $1.1 \%$ per $\mathrm{U} / \mathrm{L}$ increase in ALP concentration (OR: 1.011, 95\% CI 1.002 to 1.020 , $\mathrm{p}=0.014)$. As shown in table 2 , as a categorical variable, ALP remained associated with in-hospital mortality (OR: 3.066, 95\% CI 1.309 to $7.179, \mathrm{p}=0.010$ for Tertile 3 group vs Tertile 1 and Tertile 2 group).

Although the detailed pathophysiological association between ALP and adverse outcomes in CAD patient needs further evaluation, there are several possible explanations. First, ALP widely expresses in different tissues, and its highest concentrations were in liver, bone and kidney. ${ }^{8}$ It can hydrolyze inorganic pyrophosphate, which inhibits calcium, phosphate and hydroxyapatite crystal aggregation. $^{8}$ So, an increased ALP concentration is linked to vascular calcification. The previous study has confirmed the association between serum ALP and coronary artery calcification in CAD patients. ${ }^{9}$ Our study also found that there was a significantly higher percentage of moderate or severe calcification in the highest ALP group $(7.0 \%$ vs $5.2 \%$ and $6.6 \%, \mathrm{p}=0.034$ for Tertile 3 group vs Tertile 1 and Tertile 2 group) (table 1 ). Coronary calcification contributes to atherosclerosis, and is also a predictor of adverse cardiovascular events. ${ }^{5-7} \mathrm{Al}-$ though ACS is usually associated with non-calcified fibroatheroma, ${ }^{27}$ spot calcification still predicts atherosclerotic plaque rupture. ${ }^{28}$ Furthermore, severe coronary calcification is an independent predictor of worse prognosis in patients undergoing PCI. ${ }^{29}$ Also, coronary calcification may hamper the process of re-endothelialisation, and be a marker of extensive atherosclerotic disease. ${ }^{29}{ }^{30}$ More important, a higher serum ALP concentration is also an independent predictor of mortality, myocardial infarction, and stent thrombosis in CAD patients after PCI. ${ }^{14}$ Second, an elevated serum ALP concentration is closely associated with many cardiovascular risk factors, such as $\mathrm{C}$ reactive protein, ${ }^{31} 32$ metabolic syndrome, ${ }^{31} 32$ vitamin $\mathrm{D}$ deficiency $^{33}$ and fibroblast growth factor-23. ${ }^{34}$ It indicates that ALP may also affect the prognosis of CAD patients by interacting with cardiovascular risk factors.

Table 4 Comparisons of the predictive performance of GRACE, GRACE+ALP and ALP for the prognosis prediction

\begin{tabular}{lllllcr}
\hline & z for C-statistic & P for C-statistic & NRI & P for NRI & IDI & P for IDI \\
\hline GRACE vs ALP & 4.599 & $<0.001$ & 0.310 & $<0.001$ & 0.046 & $<0.001$ \\
GRACE+ALP vs GRACE & 0.485 & 0.628 & 0.020 & 0.630 & 0.014 \\
GRACE +ALP vs ALP & 5.411 & $<0.001$ & 0.272 & $<0.001$ & 0.056 \\
\hline
\end{tabular}

ALP, alkaline phosphatase; GRACE, Global Registry of Acute Coronary Events; IDI, integrated discrimination improvement; NRI, net reclassification improvement. 
Considering these results presented, this finding has some clinical value. First, monitoring ALP in patients with ACS may be worth, since it may help us identify the ACS patients at high risk of in-hospital death. Moreover, animal studies showed that, after inhibiting ALP, organic pyrophosphate would accumulate, and vascular smooth muscle cell calcification would be suppressed. ${ }^{35}$ This indicates that ALP may be a treatment target of coronary calcification.

This study had several limitations. First, this study was prospective and observational, so potential confounders and selection bias could not be completely adjusted. Second, data on serum parathyroid hormone level, serum vitamin D level and dietary vitamin D intake was not obtained, which might affect ALP levels. However, the previous study showed that the association between ALP and mortality was independent of parathyroid hormone or vitamin $\mathrm{D} .{ }^{23}$ Third, ALP is a membrane-bound glycoprotein (metallo-enzyme) with more than 17 circulating isoforms detectable by isoelectric focusing technique. ${ }^{36}$ We just measured tissue-non-specific ALP, but not each ALP isoforms (liver, kidney and bone), which could change the total ALP activity. However, inorganic pyrophosphate hydrolysis is a non-specific activity and can be driven by each ALP isoform. ${ }^{86}$ In addition, in this study, coronary calcification was assessed by angiography, which might underestimate the frequency and degree of coronary calcium, compared with by intravascular ultrasound. ${ }^{37}$

\section{CONCLUSION}

In patients with ACS undergoing PCI, ALP was an independent predictor of in-hospital mortality. The prognostic performance of GRACE score +ALP was similar to GRACE score, and ALP could not significantly improve the prognostic performance of GRACE score.

Acknowledgements Thank Professor Liqiang Zheng for his statistical assistance! Contributors Zhaoging Sun conceived and designed the experiments. Tongtong Yu, Yundi Jiao, Jia Song, Dongxu He, Jiake Wu, Zongyu Wen, Na Sun and Weili Duan performed the experiments. Tongtong Yu analyzed the data and wrote the paper. Zhijun Sun revised the paper. All authors had reviewed and agreed on the contents of this paper.

Funding This research project was supported by grants from the Social Development Research Program of Liaoning Province (2011225020).

Competing interests None declared.

Patient consent for publication Parental/guardian consent obtained

Ethics approval This study complies with the Declaration of Helsinki, Shengjing Hospital of China Medical University Research Ethics Committee approved the research protocol.

Provenance and peer review Not commissioned; externally peer reviewed.

Data availability statement All data relevant to the study are included in the article or uploaded as supplementary information.

Open access This is an open access article distributed in accordance with the Creative Commons Attribution Non Commercial (CC BY-NC 4.0) license, which permits others to distribute, remix, adapt, build upon this work non-commercially, and license their derivative works on different terms, provided the original work is properly cited, appropriate credit is given, any changes made indicated, and the use is non-commercial. See: http://creativecommons.org/licenses/by-nc/4.0/.

\section{REFERENCES}

1. O'Gara PT, Kushner FG, Ascheim DD, et al. 2013 ACCF/AHA guideline for the management of ST-elevation myocardial infarction: a report of the American College of cardiology Foundation/American heart association Task force on practice guidelines. J Am Coll Cardiol 2013;61:e78-140.

2. Amsterdam EA, Wenger NK, Brindis RG, et al. 2014 AHA/ACC guideline for the management of patients with non-ST-elevation acute coronary syndromes: a report of the American College of Cardiology/American heart association Task force on practice guidelines. J Am Coll Cardiol 2014;64:e139-228.

3. Roffi M, Patrono C, Collet J-P, et al. 2015 ESC guidelines for the management of acute coronary syndromes in patients presenting without persistent ST-segment elevation: Task force for the management of acute coronary syndromes in patients presenting without persistent ST-segment elevation of the European Society of cardiology (ESC). Eur Heart J 2016;37:267-315.

4. Ibanez B, James S, Agewall S, et al. 2017 ESC guidelines for the management of acute myocardial infarction in patients presenting with ST-segment elevation: the task force for the management of acute myocardial infarction in patients presenting with ST-segment elevation of the European Society of cardiology (ESC). Eur Heart $J$ 2018;39:119-77.

5. Budoff MJ, Shaw LJ, Liu ST, et al. Long-Term prognosis associated with coronary calcification: observations from a Registry of 25,253 patients. J Am Coll Cardiol 2007;49:1860-70.

6. Detrano R, Guerci AD, Carr JJ, et al. Coronary calcium as a predictor of coronary events in four racial or ethnic groups. $N$ Engl $\mathrm{J}$ Med 2008;358:1336-45

7. Van Campenhout A, Golledge J, Osteoprotegerin GJ. Osteoprotegerin, vascular calcification and atherosclerosis. Atherosclerosis 2009;204:321-9.

8. Schoppet M, Shanahan CM. Role for alkaline phosphatase as an inducer of vascular calcification in renal failure? Kidney Int 2008;73:989-91.

9. Panh L, Ruidavets JB, Rousseau H, et al. Association between serum alkaline phosphatase and coronary artery calcification in a sample of primary cardiovascular prevention patients. Atherosclerosis 2017:260:81-6.

10. Sahin I, Karabulut A, Gungor B, et al. Correlation between the serum alkaline phosphatase level and the severity of coronary artery disease. Coron Artery Dis 2014;25:349-52.

11. Tonelli M, Curhan G, Pfeffer M, et al. Relation between alkaline phosphatase, serum phosphate, and all-cause or cardiovascular mortality. Circulation 2009;120:1784-92.

12. Ndrepepa G, Xhepa E, Braun S, et al. Alkaline phosphatase and prognosis in patients with coronary artery disease. Eur J Clin Invest 2017;47:378-87.

13. Nunes JPL, Melão F, Godinho AR, et al. Plasma alkaline phosphatase and survival in diabetic patients with acute myocardial infarction. Ann Trans/ Med 2016;4.

14. Park J-B, Kang D-Y, Yang H-M, et al. Serum alkaline phosphatase is a predictor of mortality, myocardial infarction, or stent thrombosis after implantation of coronary drug-eluting stent. Eur Heart $J$ 2013;34:920-31.

15. Ndrepepa G, Holdenrieder S, Xhepa E, et al. Prognostic value of alkaline phosphatase in patients with acute coronary syndromes. Clin Biochem 2017;50:828-34.

16. Oh PC, Lee K, Kim T-H, et al. Prognostic impact of alkaline phosphatase measured at time of presentation in patients undergoing primary percutaneous coronary intervention for ST-segment elevation myocardial infarction. PLoS One 2017;12:e0171914.

17. Huseynov A, Baumann S, Becher T, et al. Liver and cholestatic parameters as prognostic biomarkers of in-hospital mace in patients with STEMI. Eur J Clin Invest 2016;46:721-9.

18. Cutlip DE, Windecker S, Mehran R, et al. Clinical end points in coronary stent trials: a case for standardized definitions. Circulation 2007;115:2344-51.

19. DeLong ER, DeLong DM, Clarke-Pearson DL. Comparing the areas under two or more correlated receiver operating characteristic curves: a nonparametric approach. Biometrics 1988;44:837-45.

20. Lemeshow S, Hosmer DW. A review of goodness of fit statistics for use in the development of logistic regression models. Am J Epidemiol. In Press 1982;115:92-106. 
21. Redelmeier DA, Bloch DA, Hickam DH. Assessing predictive accuracy: how to compare Brier scores. J Clin Epidemiol 1991;44:1141-6.

22. Pencina MJ, D' Agostino RB, D' Agostino RB, et al. Evaluating the added predictive ability of a new marker: from area under the ROC curve to reclassification and beyond. Stat Med 2008;27:157-72. Jr. Sr.,.

23. Regidor DL, Kovesdy CP, Mehrotra R, et al. Serum alkaline phosphatase predicts mortality among maintenance hemodialysis patients. J Am Soc Nephrol 2008;19:2193-203.

24. Dhingra R, Sullivan LM, Fox CS, et al. Relations of serum phosphorus and calcium levels to the incidence of cardiovascular disease in the community. Arch Intern Med 2007;167:879-85.

25. Kunutsor SK, Apekey TA, Khan H. Liver enzymes and risk of cardiovascular disease in the general population: a meta-analysis of prospective cohort studies. Atherosclerosis 2014;236:7-17.

26. Kunutsor SK, Apekey TA, Seddoh D, et al. Liver enzymes and risk of all-cause mortality in general populations: a systematic review and meta-analysis. Int J Epidemiol 2014;43:187-201.

27. Zheng B, Mintz GS, McPherson JA, et al. Predictors of plaque rupture within Nonculprit Fibroatheromas in patients with acute coronary syndromes: the prospect study. JACC Cardiovasc Imaging 2015;8:1180-7.

28. Hutcheson JD, Maldonado N, Aikawa E. Small entities with large impact: microcalcifications and atherosclerotic plaque vulnerability. Curr Opin Lipidol 2014;25:327-32.

29. Bourantas CV, Zhang Y-J, Garg S, et al. Prognostic implications of coronary calcification in patients with obstructive coronary artery disease treated by percutaneous coronary intervention: a patient-level pooled analysis of 7 contemporary stent trials. Heart 2014:100:1158-64

30. Yiu KAI-H, Wang S, MOK MO-YIN, et al. Role of circulating endothelial progenitor cells in patients with rheumatoid arthritis with coronary calcification. J Rheumatol 2010;37:529-35.

31. Cheung BMY, Ong KL, Cheung RV, et al. Association between plasma alkaline phosphatase and C-reactive protein in Hong Kong Chinese. Clin Chem Lab Med 2008;46:523-7.

32. Webber M, Krishnan A, Thomas NG, et al. Association between serum alkaline phosphatase and C-reactive protein in the United States National health and nutrition examination survey 2005-2006. Clin Chem Lab Med 2010;48:167-73.

33. Jean G, Terrat J-C, Vanel T, et al. Daily oral 25-hydroxycholecalciferol supplementation for vitamin $\mathrm{D}$ deficiency in haemodialysis patients: effects on mineral metabolism and bone markers. Nephrology Dialysis Transplantation 2008;23:3670-6.

34. Gutiérrez OM, Mannstadt M, Isakova T, et al. Fibroblast growth factor 23 and mortality among patients undergoing hemodialysis. $N$ Engl $J$ Med 2008;359:584-92.

35. Narisawa S, Harmey D, Yadav MC, et al. Novel inhibitors of alkaline phosphatase suppress vascular smooth muscle cell calcification. J Bone Miner Res 2007;22:1700-10.

36. Wallace BH, Lott JA, Griffiths J, et al. Isoforms of alkaline phosphatase determined by isoelectric focusing in patients with chronic liver disorders. Clin Chem Lab Med 1996;34:711-20.

37. Tuzcu EM, Berkalp B, De Franco AC, et al. The dilemma of diagnosing coronary calcification: angiography versus intravascular ultrasound. J Am Coll Cardiol 1996;27:832-8. 